Discussiones Mathematicae

Graph Theory 36 (2016) 565-575

doi:10.7151/dmgt.1875

\title{
ON LONGEST CYCLES IN ESSENTIALLY 4-CONNECTED PLANAR GRAPHS
}

\author{
IGOR FABRICI $^{a *}$, JOCHEN HARANT $^{b}$ \\ AND \\ StANislaV JendRol ${ }^{a}$ \\ ${ }^{a}$ Institute of Mathematics \\ P.J. Šafárik University in Košice, Slovakia \\ ${ }^{b}$ Institute of Mathematics \\ Ilmenau University of Technology, Germany
}

\begin{abstract}
A planar 3-connected graph $G$ is essentially 4-connected if, for any 3separator $S$ of $G$, one component of the graph obtained from $G$ by removing $S$ is a single vertex. Jackson and Wormald proved that an essentially 4connected planar graph on $n$ vertices contains a cycle $C$ such that $|V(C)| \geq$ $\frac{2 n+4}{5}$. For a cubic essentially 4-connected planar graph $G$, Grünbaum with Malkevitch, and Zhang showed that $G$ has a cycle on at least $\frac{3}{4} n$ vertices. In the present paper the result of Jackson and Wormald is improved. Moreover, new lower bounds on the length of a longest cycle of $G$ are presented if $G$ is an essentially 4-connected planar graph of maximum degree 4 or $G$ is an essentially 4-connected maximal planar graph.
\end{abstract}

Keywords: planar graph, longest cycle.

2010 Mathematics Subject Classification: 05C10, $05 \mathrm{C} 38$.

\section{Introduction AND Results}

We use standard notation and terminology of graph theory ([1]) and consider a finite simple 3-connected planar graph $G$ with vertex set $V(G)$ and edge set $E(G)$. Let $N(x), d(x)=|N(x)|$, and $\Delta(G)$ denote the neighborhood, the degree of

${ }^{*}$ Supported in part by Research and Development Operating Program for the project "University Science Park Technicom for innovative applications with support of knowledge technologies", code ITMS: 26220220182, co-financed from European funds. 
$x \in V(G)$ in $G$, and the maximum degree of $G$, respectively. A subset $S \subset V(G)$ is an $s$-separator of $G$ if $|S|=s$ and $G-S$ is disconnected. It is well-known that $G-S$ has exactly two components if $G$ is a 3-connected planar graph and $S$ is a 3 -separator of $G$. If $S$ is a 3-separator of a 3-connected planar graph $G$ and one component of $G-S$ is a single vertex, then $S$ is a trivial 3-separator of $G$. If $G$ is planar, 3-connected, and each 3-separator $S$ of $G$ is trivial, then $G$ is essentially 4-connected. In the present paper we are interested in the length of longest cycles of an essentially 4-connected planar graph.

Jackson and Wormald [4] proved that every essentially 4-connected planar graph on $n$ vertices contains a cycle $C$ such that $|V(C)| \geq \frac{2 n+4}{5}$. For a cubic essentially 4-connected planar graph $G$, Grünbaum and Malkevitch [3], and Zhang [8] showed that $G$ has a cycle on at least $\frac{3}{4} n$ vertices. Given a real constant $c>\frac{2}{3}$, Jackson and Wormald [4] presented an infinite family of essentially 4connected planar graphs $G$ such that $G$ does not contain a cycle on more than $c \cdot n$ vertices. This observation is even true for essentially 4 -connected maximal planar graphs. To see this, let $G^{\prime}$ be a 4 -connected maximal planar graph on $n^{\prime} \geq 6$ vertices embedded into the plane and let $G$ be obtained by inserting a new vertex into each face of $G^{\prime}$ and connecting it with all three vertices of that face by an edge. Obviously, $G$ is an essentially 4-connected maximal planar graph on $n=n^{\prime}+\left(2 n^{\prime}-4\right)$ vertices and the $2 n^{\prime}-4$ vertices in $V(G) \backslash V\left(G^{\prime}\right)$ are pairwise independent. Hence each cycle of $G$ contains at most $2 n^{\prime}=\frac{2}{3}(n+4)$ vertices. At the end of Section 2 we will show that $G$ contains a cycle on exactly $2 n^{\prime}=\frac{2}{3}(n+4)$ vertices.

It is well-known that a 3 -connected planar graph on $4 \leq n \leq 10$ vertices is Hamiltonian. It remains open whether a maximal planar (or even an arbitrary planar) essentially 4-connected graph on $n \geq 11$ vertices contains a cycle $C$ such that $|V(C)| \geq \frac{2}{3}(n+4)$.

Our results are presented in the following Theorem 1.

Theorem 1. Let $G$ be an essentially 4 -connected planar graph on $n \geq 11$ vertices and $C$ be a longest cycle of $G$. Then $|V(C)| \geq \frac{1}{2}(n+4),|V(C)| \geq \frac{3}{5} n$ if $\Delta(G)=4$, and $|V(C)| \geq \frac{13}{21}(n+4)$ if $G$ is maximal planar.

\section{ProOFs}

In the remainder of the paper we assume that $G$ is embedded into the plane. The two open sets into which a cycle $C$ of $G$ partitions the plane are the interior $\operatorname{int}(C)$ and the exterior $\operatorname{ext}(C)$ of $C$. Furthermore, let $B$ be a component of $G-V(C)$. A vertex $x \in V(C)$ is a touch vertex of $B$ if $x$ is adjacent to a vertex of $V(B)$. Note that $B$ has at least 3 touch vertices, if $G$ is a 3 -connected planar graph. In [7], Tutte proved a remarkable and famous result on cycles in 2-connected planar 
graphs implying that a 4-connected planar graph is Hamiltonian. This result has been extended several times ([5,6]). We will use the following Lemma 2 of Sanders ([5]) as a version of Tutte's result for 3-connected planar graphs.

Lemma 2. Every 3-connected planar graph $G$ with two prescribed edges $a$ and $b$ contains a cycle $C$ through $a$ and $b$ such that each component of $G-V(C)$ has exactly 3 touch vertices.

A cycle $C$ of $G$ is an outer-independent-3-cycle (OI3-cycle), if $V(G) \backslash V(C)$ is an independent set of vertices and $d(x)=3$ for all $x \in V(G) \backslash V(C)$.

Lemma 3. Let $G$ be an essentially 4-connected planar graph, and let $a$ and $b$ be non-adjacent edges of $G$. If $a$ and $b$ belong to a common face of $G$ or all end vertices of $a$ and $b$ have degree at least 4 in $G$, then $G$ contains an OI3-cycle $C$ through $a$ and $b$.

Proof. By Lemma 2, let $C$ be a cycle of $G$ through $a$ and $b$ such that each component of $G-V(C)$ has exactly three touch vertices. Since $a$ and $b$ are nonadjacent, $|V(C)| \geq 4$. We will show that $C$ is an OI3-cycle of $G$. Suppose to the contrary that $G-V(C)$ has a component $B$ with at least two inner vertices (w.l.o.g. let $V(B) \subset \operatorname{int}(C)$ ). Since $G$ is essentially 4-connected and $|V(C)| \geq 4$, the three touch vertices $y, z, u$ of $B$ separate $G$, hence they form the neighborhood of a vertex $x$ of degree 3 .

First assume that $x \in V(C)$ as shown in Figure 1 ( $C$ is the fat-drawn cycle).

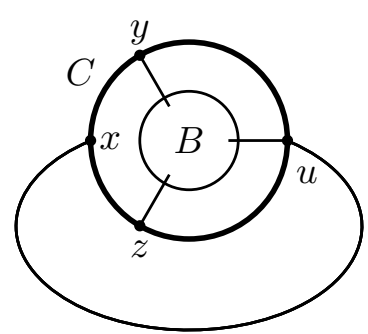

Figure 1

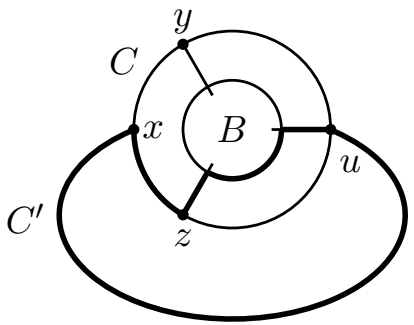

Figure 2

Let $\alpha$ be the face of $G$ containing $z, u$ and at least one vertex of $V(B)$ and let $P$ be the boundary path of $\alpha$ connecting $u$ and $z$ and containing some vertex of $V(B)$. Furthermore, let $C^{\prime}$ be the (fat-drawn) cycle with $V\left(C^{\prime}\right)=V(P) \cup\{x\}$ as shown in Figure 2. It is clear that $z$ and $u$ are the only vertices of $C^{\prime}$ which possibly have a neighbor in $\operatorname{int}\left(C^{\prime}\right) \cap V(G)$. It follows that $\operatorname{int}\left(C^{\prime}\right) \cap V(G)=\emptyset$, because otherwise $\{z, u\}$ forms a 2-separator of $G$ contradicting the 3 -connectedness of $G$. Thus $z$ and $u$ are neighbors on $C$ and, by symmetry, $y$ and $u$ are also neighbors on $C$. Consequently, $|V(C)|=4$, the edges $a$ and $b$ cannot belong to a common 
face, and one of them is incident with the vertex $x$ of degree 3 contradicting the choice of $a$ and $b$.

If $x \notin V(C)$ as shown in Figure 3, then, considering the (fat-drawn) cycles $C^{\prime \prime}$ in Figure 4 and $C^{\prime \prime \prime}$ in Figure 5, it follows that $\operatorname{int}\left(C^{\prime \prime}\right) \cap V(G)=\emptyset$ and $\operatorname{int}\left(C^{\prime \prime \prime}\right) \cap$ $V(G)=\emptyset$ with similar arguments, hence $|V(C)|=3$, also a contradiction.

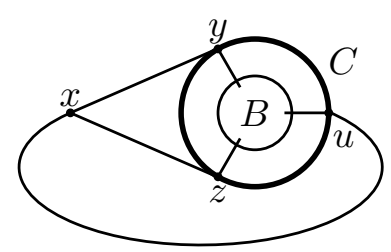

Figure 3

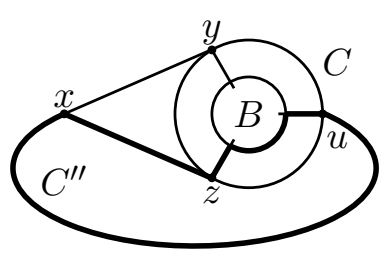

Figure 4

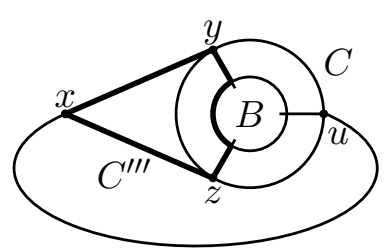

Figure 5

Consequently, $C$ is an OI3-cycle through $a$ and $b$.

Note that a Hamiltonian cycle of a graph is an OI3-cycle. Let $a=y z$ be an edge of an OI3-cycle $C$ of a graph $G$ and assume that $y$ and $z$ have a common neighbor $x \in V(G) \backslash V(C)$. Then let $C^{\prime}$ be the cycle of $G$ obtained from $C$ by replacing the edge $a$ with the path $(y, x, z)$. In this case, $a$ is an extendable edge of $C$. Note that $C^{\prime}$ is again an OI3-cycle of $G,\left|V\left(C^{\prime}\right)\right|=|V(C)|+1$, and that $C^{\prime}$ has less extendable edges than $C$. Obviously, a longest OI3-cycle of $G$ does not contain an extendable edge.

For the proof of Theorem 1 it suffices to show the following lemma.

Lemma 4. Let $G$ be an essentially 4-connected planar graph on $n \geq 11$ vertices.

(i) $G$ contains an OI3-cycle.

(ii) If $C$ is an OI3-cycle of $G$ without extendable edges, then $|V(C)| \geq \frac{1}{2}(n+4)$.

(iii) If $\Delta(G)=4$ and $C$ is an OI3-cycle of $G$, then $|V(C)| \geq \frac{3}{5} n$.

(iv) If $G$ is maximal planar and $C$ is a longest OI3-cycle of $G$, then $|V(C)| \geq$ $\frac{13}{21}(n+4)$.

Proof. If $G$ is an essentially 4-connected plane graph without vertices of degree 3 , then $G$ is even 4-connected, hence, $G$ contains a Hamiltonian cycle (Lemma 2). Since every Hamiltonian cycle is an OI3-cycle, Lemma 4(i) is true in this case. If $G$ is not maximal planar, then there exist two non-adjacent edges $a$ and $b$ of $G$ belonging to a common face, hence, by Lemma 3, Lemma 4(i) follows.

Thus, for the proof of Lemma 4(i), it remains to deal with the case that $G$ is maximal planar and contains a vertex of degree 3 . Let $a=y z$ be an edge connecting two neighbors $y$ and $z$ of a vertex $x$ of degree 3 in $G$. In this case we will show that $d(y) \geq 4, d(z) \geq 4$, and that there is an edge $b$ being nonadjacent with $a$, and with both end vertices of degree at least 4 . Consequently, the existence of an OI3-cycle in $G$ follows by Lemma 3, and Lemma 4(i) is true 
also in this case. Let $u$ be the third neighbor of $x$. The vertices $y, z, u$ form a separating 3-cycle, hence because $G$ is 3 -connected, all of them have degree at least 4. Let $w \in N(u) \backslash\{x, y, z\}$ be a fourth neighbor of $u$. If $d(u)=4$, then $\{y, z, w\}$ is a 3 -separator and both components of $G-\{y, z, w\}$ contain at least two vertices, a contradiction to the essentially 4-connectedness of $G$. It follows that $d(u) \geq 5$. Let $v \in N(u) \backslash\{x, y, z, w\}$ such that $v \in N(w)$. Since $G \not K_{4}$, vertices of degree three are not adjacent in $G$, thus one of the vertices $w$ and $v$ has degree at least four. We are done with $b=u w$ or $b=u v$, respectively, and Lemma 4(i) is completely proved.

The following Lemma 5 is proved in [2]. For completeness, we present its short proof here.

Lemma 5. If $C$ is a cycle of a plane graph $G$ on at least 4 vertices such that $\operatorname{int}(C) \cap V(G)$ is an independent set of vertices of degree 3 in $G$ and, for each edge $x y$ of $C, x$ and $y$ do not have a common neighbor in $\operatorname{int}(C) \cap V(G)$, then $|\operatorname{int}(C) \cap V(G)| \leq \frac{1}{2}(|V(C)|-4)$.

Proof. We proceed by induction on $c=|V(C)|$. If $c \leq 5$, then, obviously, $|\operatorname{int}(C) \cap V(G)|=0$. Now let $c \geq 6, d=|\operatorname{int}(C) \cap V(G)|>0$, and $\phi$ be an orientation of $C$. Consider a fixed vertex $x \in \operatorname{int}(C) \cap V(G)$ and let $x_{1}, x_{2}$, and $x_{3}$ be the neighbours of $x$ on $C$ met in this order following $\phi$. For $i=1,2,3$, let $C_{i}$ be the cycle obtained by the union of the path on $C$ from $x_{i}$ to $x_{i+1}$ following $\phi$ and the two edges $x x_{i}$ and $x x_{i+1}$ (where $x_{4}=x_{1}$ ), $c_{i}=\left|V\left(C_{i}\right)\right|$, and $d_{i}=\left|\operatorname{int}\left(C_{i}\right) \cap V(G)\right|$. Obviously, $c>c_{i} \geq 4$ and for each edge $x y$ of $C_{i}, x$ and $y$ do not have a common neighbor in $\operatorname{int}\left(C_{i}\right) \cap V(G)(i=1,2,3)$. We have $c_{1}+c_{2}+c_{3}=c+6, d_{1}+d_{2}+d_{3}=d-1$, and, by induction hypothesis, $d_{i} \leq \frac{c_{i}}{2}-2$ for $i=1,2,3$. This implies $d \leq \frac{c}{2}-2$.

To prove Lemma 4(ii), consider an OI3-cycle $C$ of $G$ without an extendable edge. Obviously, $|V(C)| \geq 4$ because $n \geq 4$. Moreover, for each edge $x y$ of $C, x$ and $y$ do not have a common neighbor in $(\operatorname{int}(C) \cup \operatorname{ext}(C)) \cap V(G)$. By Lemma 5, $|\operatorname{int}(C) \cap V(G)| \leq \frac{1}{2}(|V(C)|-4)$ and, by symmetry, $|\operatorname{ext}(C) \cap V(G)| \leq$ $\frac{1}{2}(|V(C)|-4)$. Thus $n=|V(C)|+|\operatorname{int}(C) \cap V(G)|+|\operatorname{ext}(C) \cap V(G)| \leq 2|V(C)|-4$ and Lemma 4(ii) is proved.

For the proof of Lemma 4(iii) consider an arbitrary OI3-cycle $C$ of $G$. Since $V(G) \backslash V(C)$ is an independent set and $d(x)=3$ for every $x \in V(G) \backslash V(C)$, $3(n-|V(C)|)$ equals the number $e$ of edges between $V(C)$ and $V(G) \backslash V(C)$. If $y \in V(C)$, then, because $d(y) \leq 4, y$ has at most two neighbors in $V(G) \backslash V(C)$. It follows $e \leq 2|V(C)|$ and Lemma 4(iii) is proved.

It remains to prove Lemma 4 (iv).

Let $C$ be a longest OI3-cycle of $G$. By Lemma 4(ii) and $n \geq 11$, we have $|V(C)| \geq 8$. Moreover, let $H=G[V(C)]$ be the graph obtained from $G$ by removing all vertices of degree 3 which do not lie on $C$. Obviously, $H$ is maximal 
planar and $C$ is a Hamiltonian cycle of $H$. A face $\alpha$ of $H$ is an empty face of $H$ if $\alpha$ is also a face of $G$, otherwise $\alpha$ is a non-empty face of $H$. Denote by $\mathcal{F}$ the set of empty faces of $H$. Note that every face of $G$ has at least two (of three) vertices on $C$. The three neighbors of a vertex of $V(G) \backslash V(C)$ induce a separating 3-cycle of $G$ creating the boundary of a non-empty face of $H$.

Lemma 6. Let $t=|\mathcal{F}|$ be the number of empty faces of $H$. For a positive real a, the inequalities $|V(C)| \leq$ at and $|V(C)| \geq \frac{a}{3 a-1}(n+4)$ are equivalent.

Proof. Since every face of $G$ which is not an empty face of $H$ has exactly one vertex in $V(G) \backslash V(C)$, calculating the number of faces of $G$ leads to $2 n-4=$ $t+3(n-|V(C)|)$. It follows $t=3|V(C)|-n-4$ and directly the equivalence of $|V(C)| \leq a t$ and $|V(C)| \geq \frac{a}{3 a-1}(n+4)$.

Using Lemma 6, it suffices to prove $|V(C)| \leq \frac{13}{18} t$.

Let $H_{1}$ and $H_{2}$ be the spanning subgraphs of $H$ consisting of the cycle $C$ and of its chords lying in the interior and in the exterior of $C$, respectively. Note that $E\left(H_{1}\right) \cap E\left(H_{2}\right)=E(C)$ and $H_{1}$ and $H_{2}$ are maximal outerplanar graphs.

An empty face $\varphi$ of $H$ is a $j$-face if exactly $j$ of its three incident edges belong to $E(C)$. Since $|V(C)| \geq 8$, it follows $j \in\{0,1,2\}$ for any $j$-face $\varphi$ of $H$. Note that $C$ and a non-empty face of $H$ do not have an edge in common because otherwise such an edge would be an extendable edge of $C$ in $G$.

Since $C$ does not contain extendable edges, every face of $H$ incident with an edge of $C$ is an empty face. An edge $e$ of $C$ incident with the faces $\varphi$ and $\psi$ is a $(j, k)$-edge for $1 \leq j, k \leq 2$, if $\varphi$ is a $j$-face and $\psi$ is a $k$-face.

For every edge $e \in E(C)$ we define the weight $w_{0}(e)=1$. Obviously, $\sum_{e \in E(C)} w_{0}(e)=|V(C)|$.

\section{First redistribution of weights}

If $x, y$, and $z$ are the vertices incident with a face $\varphi$ of $H$, then we write $\varphi=$ $[x, y, z]$. Let $(u, x, y, v)$ be a subpath of $C, x y$ be a $(2,2)$-edge of $C$, and $\alpha=$ $[u, x, y]$ and $\sigma=[x, y, v]$ be two adjacent 2-faces of $H$. Moreover, let $\beta$ and $\tau$ be the faces of $H$ incident with $u y$ and $x v$ and distinct from $\alpha$ and $\sigma$, respectively (see Figure 6 ). The cycle $\widetilde{C}$ obtained from $C$ by replacing the path $(u, x, y, v)$ by the path $(u, y, x, v)$ is also a longest OI3-cycle of $G$, hence both $u y$ and $x v$ are not extendable edges of $\widetilde{C}$ and therefore $\beta$ and $\tau$ are also empty faces of $H$.

The weight of all edges of $C$ will be completely redistributed to empty faces of $H$ by the following rules.

Rule R1. A $(2,2)$-edge $x y$ of $C$ (Figure 6) sends weight $\frac{1}{3}$ to both incident 2-faces $\alpha$ and $\sigma$ and weight $\frac{1}{6}$ to $\beta$ (through the edge $u y$ ) and to $\tau$ (through the edge $x v$ ). 
Rule R2. A (1,2)-edge of $C$ sends weight $\frac{2}{3}$ to the incident 1 -face and weight $\frac{1}{3}$ to the incident 2 -face.

Rule R3. A $(1,1)$-edge of $C$ sends weight $\frac{1}{2}$ to both incident 1 -faces.

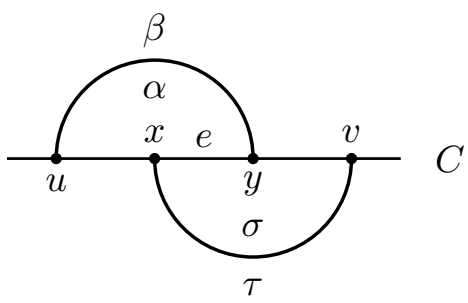

Figure 6

For an empty face $\varphi$, let $w_{1}(\varphi)$ be the total weight obtained by $\varphi$ (in first redistribution). Obviously, $\sum_{\varphi \in \mathcal{F}} w_{1}(\varphi)=|V(C)|$.

Every empty face gets weight from (or through) at most two of its three incident edges (otherwise $|V(C)| \leq 6$, a contradiction). An empty face $\varphi$ of $H$ is good if $w_{1}(\varphi) \leq \frac{2}{3}$, otherwise it is bad.

Every 2 -face $\varphi$ gets weight only by rules R1 or R2, thus $w_{1}(\varphi) \leq \frac{1}{3}+\frac{1}{3}=\frac{2}{3}$ and $\varphi$ is good.

A 0 -face $\varphi$ can get weight only by rule R1. It can get weight $\frac{1}{6}$ from two distinct edges of $C$ through the same incident edge, thus $w_{1}(\varphi) \leq\left(\frac{1}{6}+\frac{1}{6}\right)+$ $\left(\frac{1}{6}+\frac{1}{6}\right)=\frac{2}{3}$ and $\varphi$ is good.

Every 1 -face $\varphi$ gets weight $\frac{2}{3}$ (by R2) or weight $\frac{1}{2}$ (by R3) from the incident edge lying on $C$. Furthermore, $\varphi$ can get weight also through one of the remaining two incident edges (by R1). Thus $w_{1}(\varphi) \leq \frac{2}{3}+\left(\frac{1}{6}+\frac{1}{6}\right)=1$. Moreover, if $\varphi$ is bad, then $w_{1}(\varphi)=\frac{5}{6}$ or $w_{1}(\varphi)=1$.

Now we describe all possible neighborhoods of bad faces.

Lemma 7. Let $\beta \in F\left(H_{i}\right), i \in\{1,2\}$, be a bad face of $H$ and let $\alpha$ and $\gamma$ be the two faces of $H_{i}$ adjacent to $\beta$, where $\alpha$ is a 2 -face of $H$. The face $\beta$ is of one of the following four types (Figure 7 ):

(B1) $w_{1}(\beta)=\frac{5}{6}$ and $\gamma$ is an empty face,

(B2) $w_{1}(\beta)=1$ and $\gamma$ is an empty 0-face,

(B3) $w_{1}(\beta)=1$ and $w_{1}(\gamma)=\frac{1}{2}$,

(B4) there is a 2-face $\sigma$ of $H_{3-i}$ adjacent (in $H$ ) to $\alpha, \beta$, and $\tau$, where $\tau$ is an empty 0 -face of $H$.

Proof. If $\beta \in F\left(H_{i}\right), i \in\{1,2\}$, is a bad face of $H$, then there is a 2-face $\alpha$ of $H_{i}$ adjacent to $\beta$. Let $\gamma(\gamma \neq \alpha)$ be the second face of $H_{i}$ adjacent to $\beta$ (Figure 8). 
(B1)

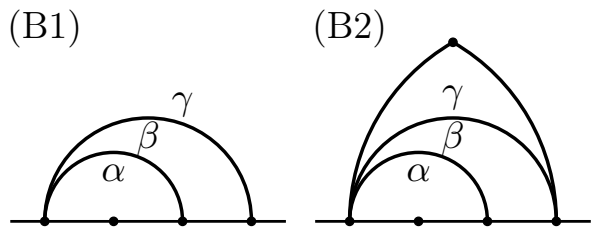

(B3)

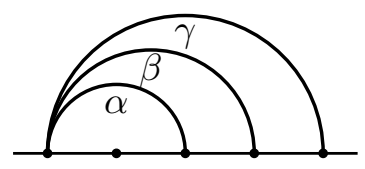

(B4)

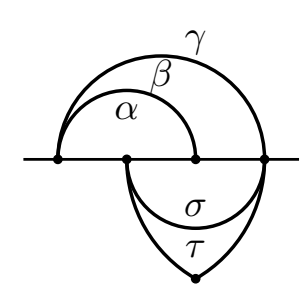

Figure 7

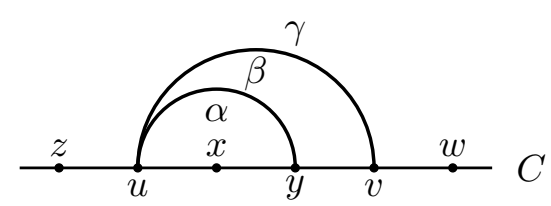

Figure 8

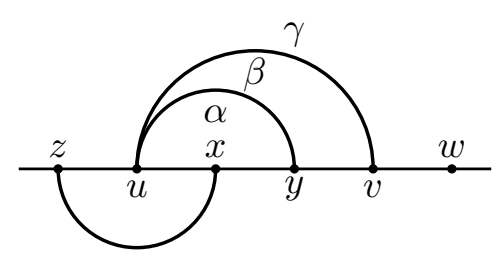

Figure 9

Case 1. Let $w_{1}(\beta)=\frac{5}{6}$ and $u x$ be a $(2,2)$-edge (i.e., $z x \in E\left(H_{3-i}\right)$, see Figure 9$)$. The cycle $\widetilde{C}$ obtained from $C$ by replacing the path $(z, u, x, y, v)$ by the path $(z, x, y, u, v)$ is a longest OI3-cycle of $G$ and contains the edge $u v$, thus $\gamma$ is an empty face of $H$ (and $\beta$ is of type B1).

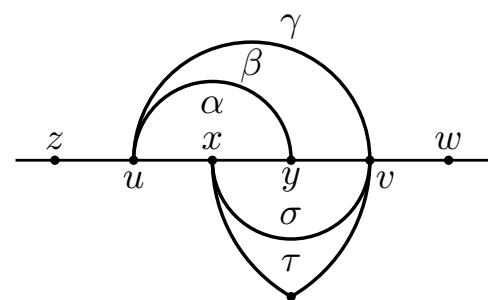

Figure 10

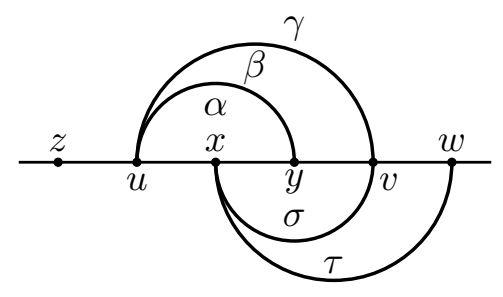

Figure 11

Case 2. Let $w_{1}(\beta)=\frac{5}{6}$ and $x y$ be a $(2,2)$-edge (i.e., $\left.x v \in E\left(H_{3-i}\right)\right)$. The face $\sigma=[x, y, v]$ is a 2-face of $H_{3-i}$. Let $\tau(\tau \neq \sigma)$ be the second face of $H_{3-i}$ incident with $x v$. Since $|V(C)| \geq 8$, it follows $u \neq w$, hence $\tau$ cannot be a 2-face of $H_{3-i}$.

Case 2.1. If $\tau$ is a 0 -face (Figure 10), then the cycle $\widetilde{C}$ obtained from $C$ by replacing the path $(u, x, y, v)$ by the path $(u, y, x, v)$ is a longest OI3-cycle of $G$ and contains the edge $x v$, thus $\tau$ is an empty face of $H$ (and $\beta$ is of type B4).

Case 2.2. If $\tau$ is a 1-face (Figure 11), then $\tau=[x, v, w]$ (since $u v \in E\left(H_{i}\right) \backslash$ $E(C), u v$ is not an edge of $\left.H_{3-i}\right)$. The cycle $\widetilde{C}$ obtained from $C$ by replacing the path $(u, x, y, v, w)$ by the path $(u, v, y, x, w)$ is a longest OI3-cycle of $G$ and contains the edge $u v$, thus $\gamma$ is an empty face of $H$ (and $\beta$ is of type B1). 


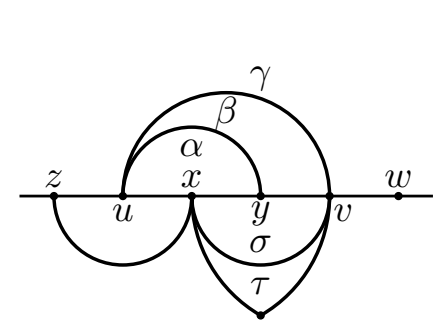

Figure 12

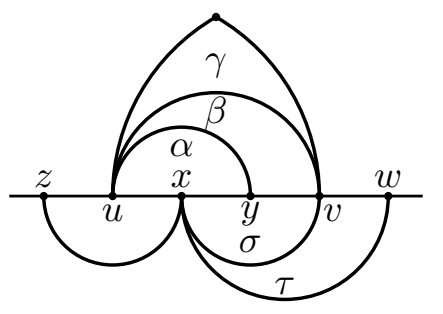

Figure 13

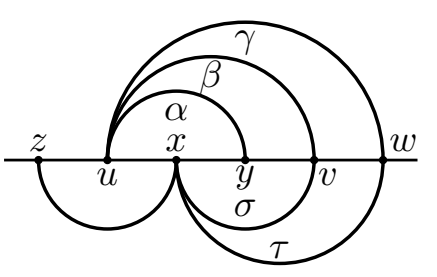

Figure 14

Case 3. Let $w_{1}(\beta)=1$. Now both $u x$ and $x y$ are $(2,2)$-edges (i.e., $z x, x v \in$ $\left.E\left(H_{3-i}\right)\right)$. The face $\sigma=[x, y, v]$ is a 2-face of $H_{3-i}$. Let $\tau(\tau \neq \sigma)$ be the second face of $H_{3-i}$ incident with $x v$. Again, $\tau$ cannot be a 2 -face of $H_{3-i}$ and we consider two subcases.

Case 3.1. If $\tau$ is a 0 -face (see Figure 12, possibly $\tau=[z, x, v]$ ), then, for a similar reason as in Case 2.1, $\tau$ is an empty face of $H$ (and $\beta$ is of type B4).

Case 3.2. If $\tau$ is a 1 -face, then $\tau=[x, v, w]$. Since $|V(C)| \geq 8$, it follows $z \neq w$, hence $\gamma$ is not a 2 -face of $H_{i}$. We consider the last two subcases.

Case 3.2.1. If $\gamma$ is a 0 -face (see Figure 13), then, for a similar reason as in Case $1, \gamma$ is an empty face of $H$ (and $\beta$ is of type B2).

Case 3.2.2. If $\gamma$ is a 1 -face, then $\gamma \neq[z, u, v]$ (otherwise $\{z, x, v\}$ is a nontrivial 3-separator, a contradiction). Thus $\gamma=[u, v, w]$ (see Figure 14) and $v w$ is a $(1,1)$-edge (and $\beta$ is of type B3).

For a better overview, we list the current weights of all faces considered in Lemma 7:

(B1) $w_{1}(\alpha)=\frac{2}{3}, w_{1}(\beta)=\frac{5}{6}$, and $w_{1}(\gamma) \leq \frac{2}{3}$;

(B2) $w_{1}(\alpha)=\frac{2}{3}, w_{1}(\beta)=1$, and $w_{1}(\gamma) \leq \frac{1}{3}$, because $\gamma$ obtains no weight through its common edge with $\beta$ and at most $\frac{1}{6}+\frac{1}{6}$ through at most one of its remaining two edges;

(B3) $w_{1}(\alpha)=\frac{2}{3}, w_{1}(\beta)=1$, and $w_{1}(\gamma)=\frac{1}{2}$;

(B4) $w_{1}(\alpha)=\frac{2}{3}, \frac{5}{6} \leq w_{1}(\beta) \leq 1, w_{1}(\sigma)=\frac{2}{3}$, and $w_{1}(\tau) \leq \frac{1}{2}$, because $\tau$ obtains weight $\frac{1}{6}$ through its common edge with $\sigma$ and at most $\frac{1}{6}+\frac{1}{6}$ through at most one of its remaining two edges.

\section{Second redistribution of weights}

The weight of all bad faces exceeded $\frac{13}{18}$ will be redistributed to good faces in their neighborhoods.

Rule R4. A bad face $\beta$ of type B1 sends weight $\frac{1}{18}$ to $\alpha$ and to $\gamma$ (through the common edge).

Rule R5. A bad face $\beta$ of type B2 or B3 sends weight $\frac{1}{18}$ to $\alpha$ and weight $\frac{2}{9}$ to $\gamma$ (through the common edge). 
Rule R6. A bad face $\beta$ of type B4 sends weight $\frac{1}{18}$ to $\alpha$ and to $\sigma$ (through the common edge) and the weight $\frac{1}{6}$ to $\tau$ (through the edge $x v$, see Figure 10).

For an empty face $\varphi$, let $w_{2}(\varphi)$ be the total weight of $\varphi$ (after second redistribution). Obviously, $\sum_{\varphi \in \mathcal{F}} w_{2}(\varphi)=\sum_{\varphi \in \mathcal{F}} w_{1}(\varphi)=|V(C)|$.

A bad face $\varphi$ of type B1 sends weight $2 \times \frac{1}{18}$ to $\operatorname{good}$ faces, thus $w_{2}(\varphi)=$ $\frac{5}{6}-2 \times \frac{1}{18}=\frac{13}{18}$. A bad face $\varphi$ of type B2 or B3 sends weight $\frac{1}{18}+\frac{2}{9}$ to good faces, thus $w_{2}(\varphi)=1-\frac{1}{18}-\frac{2}{9}=\frac{13}{18}$. Finally, a bad face $\varphi$ of type B4 sends weight $2 \times \frac{1}{18}+\frac{1}{6}$ to good faces, thus $w_{2}(\varphi) \leq 1-2 \times \frac{1}{18}-\frac{1}{6}=\frac{13}{18}$.

If a 2 -face $\varphi$ gets weight by the rules $\mathrm{R} 4, \mathrm{R} 5$, or $\mathrm{R} 6$, then either by exactly one of the rules $\mathrm{R} 4$ and $\mathrm{R} 5$ ( $\varphi=\alpha$ is adjacent to a 1 -face $\beta$ in this case) or by R6 ( $\varphi=\sigma$ is adjacent to a 0 -face $\tau$ in this case). Thus $w_{2}(\varphi) \leq \frac{2}{3}+\frac{1}{18}=\frac{13}{18}$.

A good 1-face $\varphi$ has at most one adjacent bad face (otherwise $|V(C)| \leq 7$ by Lemma 7, a contradiction). If $w_{1}(\varphi)=\frac{1}{2}$, then $w_{2}(\varphi) \leq \frac{1}{2}+\frac{2}{9}=\frac{13}{18}$ (by R5). If $w_{1}(\varphi)=\frac{2}{3}$, then $w_{2}(\varphi) \leq \frac{2}{3}+\frac{1}{18}=\frac{13}{18}$ (by R4).

A 0 -face $\varphi$ gets through at least one of its incident edges no weight in first redistribution (1RD) and also in second redistribution (2RD). Let $e$ be an edge incident with $\varphi$. If $\varphi$ gets weight $\frac{2}{9}$ through $e$ (by $\mathrm{R} 5$ ) in $2 \mathrm{RD}$, then $\varphi$ obtained no weight through $e$ in $1 \mathrm{RD}$. If $\varphi$ gets weight $\frac{1}{6}$ through $e$ (by R6) in $2 \mathrm{RD}$, then $\varphi$ has already obtained weight $\frac{1}{6}$ through $e$ in 1RD. Finally, if $\varphi$ gets no weight through $e$ in $2 \mathrm{RD}$, then $\varphi$ has obtained weight at most $\frac{1}{3}$ through $e$ in $1 \mathrm{RD}$. Thus $\varphi$ obtain through $e$ weight at most $\frac{1}{3}$ (in $1 \mathrm{RD}$ and $2 \mathrm{RD}$ in total) and $w_{2}(\varphi) \leq \frac{1}{3}+\frac{1}{3}=\frac{2}{3}$ follows. Thus, Lemma 4 is completely proved.

It remains to show that the essentially 4-connected maximal planar graph $G$ on $n=n^{\prime}+\left(2 n^{\prime}-4\right)$ vertices constructed in Section 1 from the 4-connected maximal planar graph $G^{\prime}$ on $n^{\prime} \geq 6$ vertices contains a cycle on exactly $2 n^{\prime}$ vertices. To see this, let $a$ and $b$ be two adjacent edges of $G^{\prime}$ which do not belong to a common face of $G^{\prime}$. Note that $a$ and $b$ exist since $n \geq 6$ implies that each vertex of $G^{\prime}$ has degree at least 4 . Consider a Hamiltonian cycle $C^{\prime}$ of $G^{\prime}$ through $a$ and $b$ (apply Lemma 2). Let $a=e_{1}, e_{2}, \ldots, e_{n^{\prime}-1}, e_{n^{\prime}}=b$ be the edges of $C^{\prime}$ met in this order along $C^{\prime}$. For $j=1, \ldots, n^{\prime}$, consider the common neighbors $x_{j} \in\left(V(G) \backslash V\left(G^{\prime}\right)\right) \cap \operatorname{int}\left(C^{\prime}\right)$ and $y_{j} \in\left(V(G) \backslash V\left(G^{\prime}\right)\right) \cap \operatorname{ext}\left(C^{\prime}\right)$ of the end vertices $u_{j}$ and $v_{j}$ of $e_{j}$. It is easy to see that the vertices in $\left\{x_{1}, \ldots, x_{n^{\prime}}, y_{1}, \ldots, y_{n^{\prime}}\right\}$ are pairwise distinct (if $n^{\prime}$ is odd, then note that $a$ and $b$ do not belong to a common face of $G^{\prime}$ ). Eventually, let the cycle $C$ of $G$ be obtained by replacing $e_{j}$ in $C^{\prime}$ with the path $\left(u_{j}, x_{j}, v_{j}\right)$ if $j$ is odd and $\left(u_{j}, y_{j}, v_{j}\right)$ if $j$ is even $\left(j=1, \ldots, n^{\prime}\right)$.

\section{REFERENCES}

[1] J.A. Bondy and U.S.R. Murty, Graph Theory (Springer, 2008).

[2] I. Fabrici, J. Harant and S. Jendrol, Paths of low weight in planar graphs, Discuss. Math. Graph Theory 28 (2008) 121-135.

doi:10.7151/dmgt.1396 
On Longest Cycles in Essentially 4-Connected Planar Graphs 575

[3] B. Grünbaum and J. Malkevitch, Pairs of edge-disjoint Hamilton circuits, Aequationes Math. 14 (1976) 191-196.

doi:10.1007/BF01836218

[4] B. Jackson and N.C. Wormald, Longest cycles in 3-connected planar graphs, J. Combin. Theory Ser. B 54 (1992) 291-321. doi:10.1016/0095-8956(92)90058-6

[5] D.P. Sanders, On paths in planar graphs, J. Graph Theory 24 (1997) 341-345. doi:10.1002/(SICI)1097-0118(199704)24:4<341::AID-JGT6>3.0.CO;2-O

[6] C. Thomassen, A theorem on paths in planar graphs, J. Graph Theory 7 (1983) $169-176$. doi:10.1002/jgt.3190070205

[7] W.T. Tutte, A theorem on planar graphs, Trans. Amer. Math. Soc. 82 (1956) 99-116. doi:10.1090/S0002-9947-1956-0081471-8

[8] C.-Q. Zhang, Longest cycles and their chords, J. Graph Theory 11 (1987) 521-529. doi:10.1002/jgt.3190110409

Received 16 June 2015

Revised 23 September 2015

Accepted 23 September 2015 